\title{
Impact of a low protein diet during pregnancy in sheep on insulin and vascular endothelial growth factor signalling
}

\author{
Louise J. Lloyd \& David S. Gardner
}

School of Veterinary Medicine and Science, University of Nottingham, Sutton Bonington Campus, LE12 5RD, UK

Email: louise.lloyd@nottingham.ac.uk

\section{INTRODUCTION}

Maternal malnutrition leads to offspring with a greater risk of developing chronic kidney disease

We have previously shown that a maternal low protein diet impacts upon early developmental processes in the fetal kidney to reduce nephron number and renal function in the mature kidneya

This study considers insulin and vascular endothelial growth factor (VEGF) signalling in the kidney and liver.

\section{METHODS}

Pregnant twin-bearing ewes were fed a control diet providing adequate dietary protein (control, $C ; n=15)$, or a low protein diet ( $L P, n=16$ ) from the day of artificial insemination to 0.44 gestation when ewes and fetuses were euthanised. A further cohort of fetuses continued to full term ( $C, n=6 ; L P, n=7)$ and were killed at two years of age.

From each fetus or adult sheep tissues were fixed in $4 \%$ paraformaldehyde or snap-frozen.

Gene expression was quantified using Real-Time PCR, and protein expression by immunohistochemistry.

Data are mean \pm e.s.e. and were analysed by linear mixed-effects models (Genstat v14, VSNi, UK).

\section{RESULTS}

In the fetal kidney VEGFA protein was reduced by $30-40 \%$ in LP vs. controls (Figure 1 ). In the adult kidney, the situation is reversed, with VEGF protein significantly higher in LP animals compared with controls. This likely reflects the differing roles of VEGFA during development (angiogenesis) and adulthood (maintenance and repair of blood vessels).
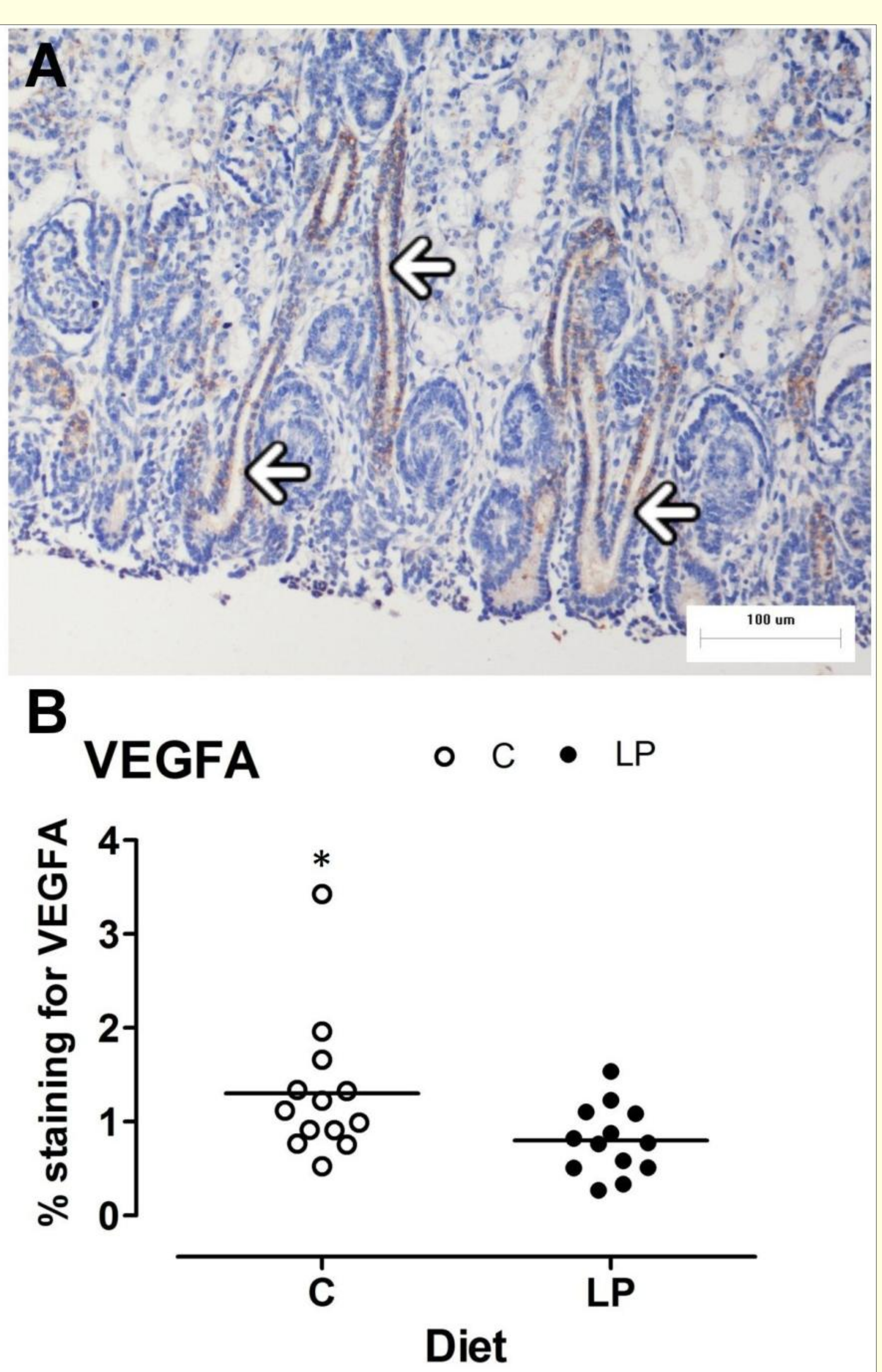

Figure 1: Fetal kidney stained for VEGFA. A representative micrograph with white arrows indicating developing collecting ducts. $\mathrm{B}$, Quantification of VEGFA staining. C, control protein; LP, low protein; VEGFA, vascular endothelial growth factor $A, * P<0.05$
- In the fetal and adult kidney VEGFA gene expression reflects the changes seen at the protein level. In contrast, in the fetal liver, VEGFA mRNA was increased 3-fold in LP fetuses vs. controls, but was reduced in LP adults; Figure 2. Thus a low protein diet during pregnancy affects VEGFA levels in the fetus and later in life in adulthood, however the effects are organ-specific.

- As adults, the LP exposed animals had hepatic insulin resistance (Figure 3), and downregulated insulin-sensitive genes such as Akt2, IRS-1 and FOXO1 in the liver. However, in the adult kidneys, at this stage there were no significant reductions in the insulin pathway genes in the LP group compared with controls. Therefore insulin sensitivity appears to be organ-specific.

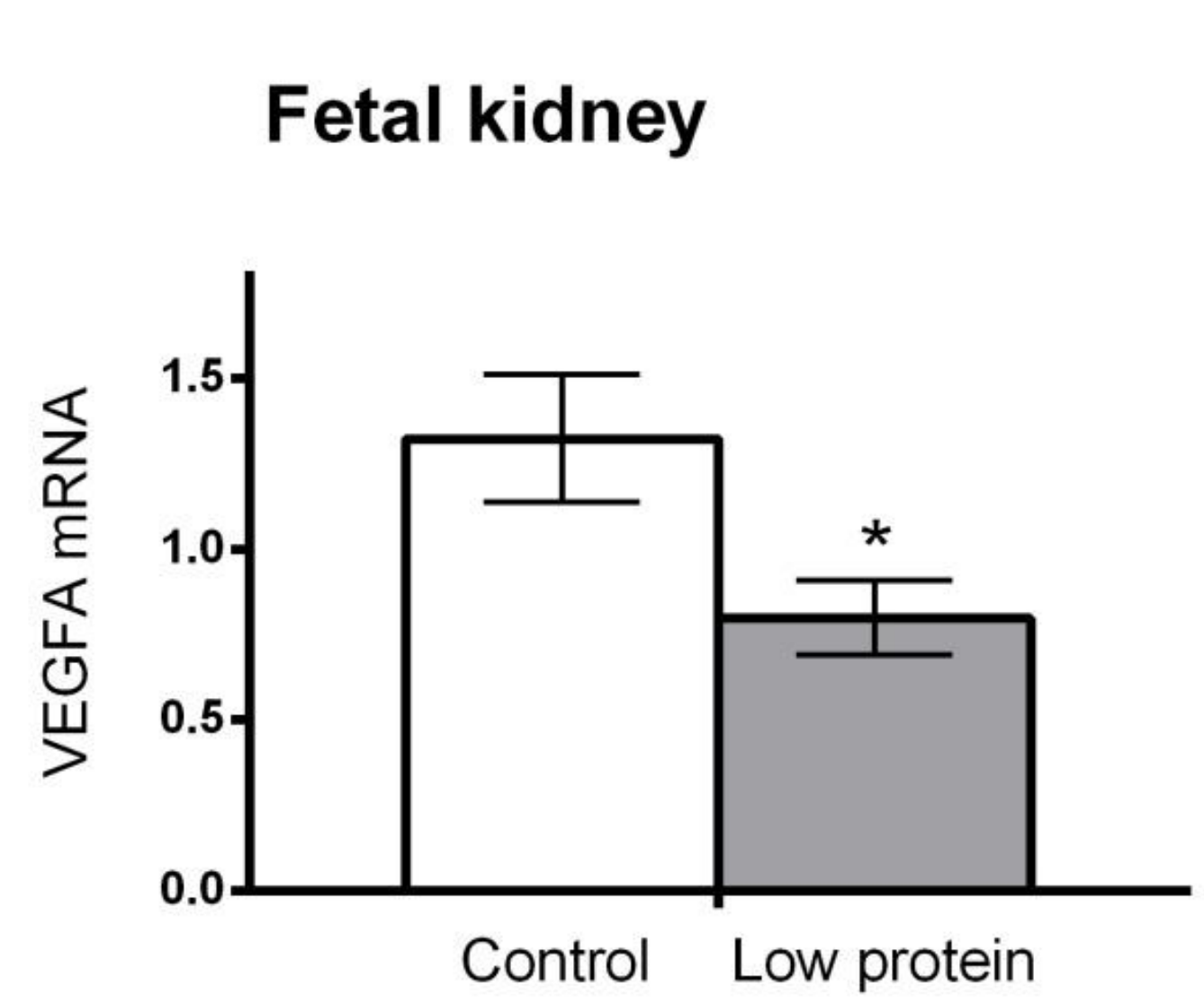

Adult kidney

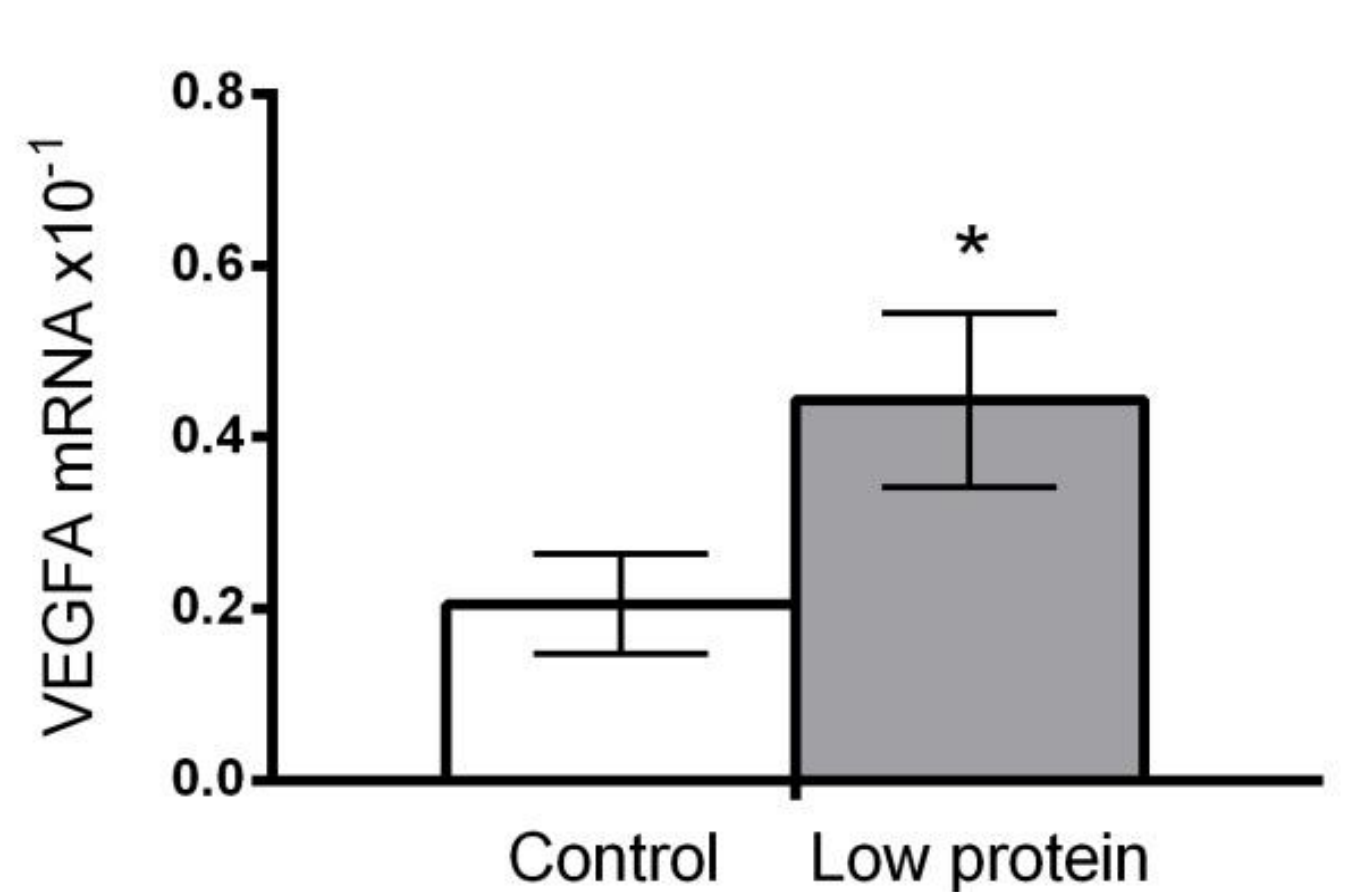

Figure 2: VEGFA mRNA expression in fetal kidney and liver, and adult kidney and liver. $* P<0.05 ; * * * P<0.001$.



Adult Liver

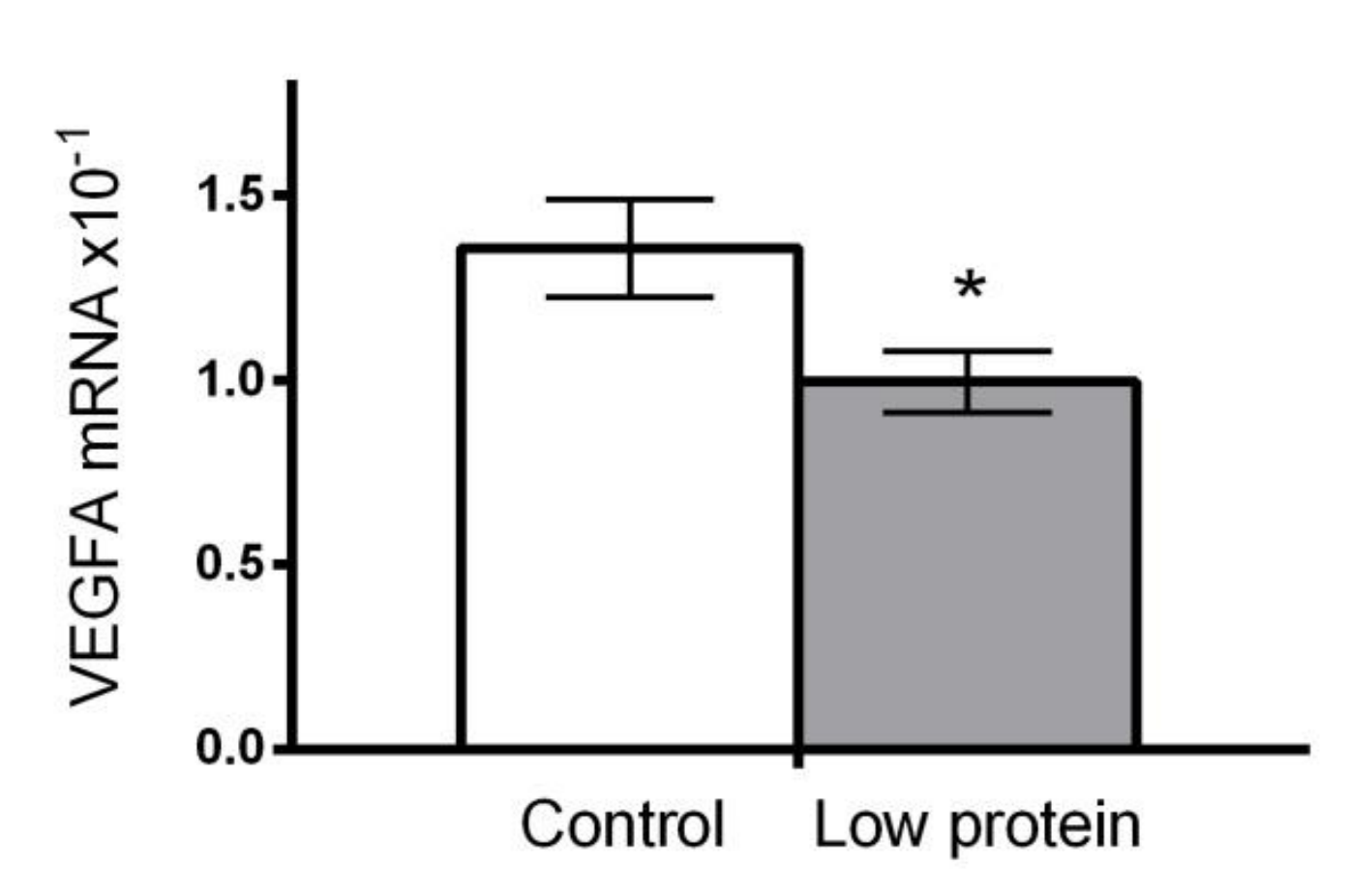



Figure 3: HOMA-IR for two-year old sheep fed either a control diet $(n=6)$ or low protein diet $(\mathrm{n}=7)$ in utero. $* * P<0.01$; HOMA-IR, homeostatic model assessment of insulin resistance.

This work was funded by a British Heart Foundation PhD

studentship, and a Society for

Endocrinology Early Career Grant.

\section{CONCLUSION}

A low protein diet during pregnancy leads to hepatic insulin resistance and effects on VEGF in both the kidney and liver. However, these effects are organ-specific and do not translate to other organs. 